\title{
Investment under Uncertainty and Policy Change*
}

\author{
Grzegorz Pawlina ${ }^{\dagger}$ and Peter M. Kort ${ }^{\ddagger}$
}

July 6,2003

\begin{abstract}
In this paper the impact of a policy change on the investment behavior of the firm is studied in an asymmetric information setting. The policy change occurs when a stochastic process describing the state of the economic environment reaches a certain trigger. Asymmetric information is modeled here by imposing that the firm does not know this trigger but only its probability distribution. Consequently, both the firm's conjecture concerning the trigger value as well as the precision of this conjecture serve as input parameters. We derive the optimal investment rule maximizing the value of the firm. We show that the impact of trigger value uncertainty is non-monotonic: the investment threshold decreases with the trigger value uncertainty for low levels of uncertainty, while the reverse is true for high uncertainty levels. Moreover, we provide results concerning the valuation of the firm's investment opportunity. Finally, based on the firm's value-maximizing behavior, policy implications for the authority are presented.

Keywords: investment under uncertainty, real options, policy change, asymmetric information
\end{abstract}

JEL classification: C61, D81, G31

${ }^{*}$ This research was undertaken with support from the European Union's Phare ACE Programme 1997. The content of the publication is the sole responsibility of the authors and in no way represents the views of the Commission or its services. The authors would like to thank Tom Berglund, Ulrich Hege, Kuno Huisman, and an anonymous referee for helpful comments and suggestions. All remaining errors are ours.

${ }^{\dagger}$ Corresponding author. Department of Econometrics \& Operations Research, and CentER, Tilburg University, P.O. Box 90153, 5000 LE Tilburg, the Netherlands, email: g.pawlina@uvt.nl, phone: + 31134663178 , fax: + 31134663280 .

${ }^{\ddagger}$ Department of Econometrics \& Operations Research, and CentER, Tilburg University, the Netherlands, and Department of Economics, University of Antwerp, Belgium, email: kort@uvt.nl. 


\section{Introduction}

Corporate investment opportunities may be represented as a set of (real) options to acquire productive assets. In the literature it is widely assumed that the present values of cash flows generated by these assets are uncertain and that their evolution can be described by a stochastic process. Consequently, identification of the optimal exercise strategies for real options plays a crucial role in capital budgeting and in the maximization of a firm's value.

So far, the real options literature provides relatively little insight into the impact of structural changes of the economic environment on the investment decisions of a firm. The existing papers (excellent surveys of those are provided in Dixit and Pindyck, 1996, Lander and Pinches, 1998, and Schwartz and Trigeorgis, 2001) mainly consider continuous changes in the values of relevant variables. Most of the time, this results in the assumption that the entire uncertainty in the economy can be described by a geometric Brownian motion.

In case of structural changes it is more realistic to model an economic variable as a process that makes infrequent but discrete jumps. ${ }^{1}$ In such cases use is made of a Poisson (jump) process. However, within such a framework the implicit assumption is made that the firm has virtually no information about the mechanisms governing the shocks in the economy. It is natural to assume that the firm can to some extent assess the precision of its conjecture concerning the moment of change, i.e. that it knows the variance of the estimate of timing of the future event. A Poisson based approach does not allow for including this type of uncertainty in the analysis since it entails a single parameter characterizing the arrival rate of the jump. Consequently, such a modelling approach lacks degrees of freedom necessary for capturing both the expectation and the precision of this expectation.

We propose a method to model the impact of a policy change on the investment strategy of the firm that takes into account the precision of information concerning the policy change possessed by the firm while making the investment decision. In our approach the expectation of the moment of the change as well as its variance serve as input parameters. We model the policy change as being triggered by a sufficiently high realization of a stochastic process related to the value of the investment opportunity. The policy change results in an upward jump in the (net) investment cost. The firm is not aware of the exact value of the trigger but it knows the probability distribution underlying the trigger. Taking into account consistent authority behavior, the firm knows that a jump will not occur as long as the current value of the variable remains below the maximum that this variable has attained in the past. When the underlying variable reaches a new maximum and still the jump does not occur, the firm updates its conjecture about the value of the barrier.

An interesting example of a structural change in the economy has been provided by Hassett and Metcalf (1999), who analyze how an expected reduction in the investment tax

\footnotetext{
${ }^{1}$ For instance, recent tax debates both in the US and across Europe constitute a significant source of uncertainty associated with discountinuous changes in the economic environment.
} 
credit influences capital investment behavior. ${ }^{2}$ In their setting a Poisson process describes the changes in the tax regime that affect the value of the investment opportunity. The framework of our paper coincides with Hassett and Metcalf (1999) in the sense that also here the firm expects a policy change but it does not know the exact moment at which it will occur. However, the firm typically expects the reduction to be imposed when the economy is booming and an active pro-investment policy is no longer needed or desired. Our framework takes this aspect into account, and this is exactly how Hassett and Metcalf (1999) is extended. Applying the Poisson based methodology is equivalent to assuming that it is time itself and not the state of economic environment that governs the change. As we argue, the moment of the reduction depends on the state of the economy. This is in contrast with the models based on the Poisson process where the probability of the change is constant over time. ${ }^{3,4}$

Consequently, our objective is to determine the optimal timing of an irreversible investment when the investment cost is subject to change and the firm has incomplete information about the moment of the change. It is clear that the value of the investment opportunity drops to zero at the moment that the investment cost jumps to infinity. However, we consider a situation where the cost of investment can be finite after the occurrence of an upward jump. In this respect this work generalizes Berrada (1999), Schwartz and Moon (2000), and Lambrecht and Perraudin (2003), in which the value of the project drops to zero at the unknown point of time.

Our main results are the following. An equation is derived that implicitly determines the value of the project at which the firm is indifferent between investing and refraining from investment. This value is the optimal investment threshold and it is shown that this threshold is decreasing in the hazard rate of the cost-increase trigger. We show that for a given value of the project, the hazard rate first increases and then decreases with trigger value uncertainty. It turns out that this non-monotonic relationship is crucial for our main finding. This main finding is that the investment threshold decreases with the trigger value uncertainty when the uncertainty is low, while it increases with uncertainty for high uncertainty levels. The implication is that an intermediate uncertainty level exists at which the threshold value is

\footnotetext{
${ }^{2}$ Other contributions concerning the impact of policy risk on irreversible investment include Altug et al. (2001), and Panteghini and Scarpa (2003).

${ }^{3}$ Hassett and Metcalf (1999) try to correct this by letting the arrival rate depend on the output price. But still it is then possible that an investment subsidy is reduced for low output prices, while the subsidy was maintained under high output prices. This kind of inconsistency in the authority's behavior is no longer possible under our approach.

${ }^{4}$ There are other economic situations in which it is realistic to impose a certain relationship between the occurrence of the shock and the state of the economy. A foreign direct investment decision to purchase a privatized enterprise where the local government may increase the offering price after the performance of the enterprise improves, can also be perceived as an option with an embedded risk of an increase in the strike price. A non-exclusive investment opportunity for which a competitive bid can be expected can serve as another example.
} 
lowest, i.e. the firm will invest at the earliest instance exactly in this situation. Hence, for a policy maker interested in accelerating investment, an optimal (strictly positive) level of the trigger value uncertainty can be identified which is the level corresponding to the minimal investment threshold. Furthermore, we show that the relationship between the value of the investment opportunity and policy uncertainty is non-monotonic.

In Section 2 we present the model of investment with the jump in the investment cost that results from a policy change. Section 3 provides the major results and Section 4 contains a numerical valuation of the investment opportunity and analyzes the probability of investment before the policy change is imposed. In Section 5 we present the implications of our model for the authority that considers an investment tax credit policy change, and Section 6 concludes.

\section{The Model}

It is assumed that the value of the investment project follows a geometric Brownian motion

$$
d V(t)=\alpha V(t) d t+\sigma V(t) d w(t)
$$

where parameter $\alpha$ denotes the deterministic drift parameter, $\sigma$ is the instantaneous standard deviation, and $d w$ is the increment of a Wiener process. The riskless rate is $r$ and it holds that $\alpha<r$. The firm is risk-neutral and maximizes the value of the investment option, $F(V)$. If the value of the investment project reaches a critical level, a change in the value of a certain policy instrument is imposed and, as a result, an effective increase in the investment cost occurs. ${ }^{5}$ This instrument can be interpreted, among others, as a reduction in the investment tax credit, an increase in the cost of capital via lending rates or an increase in the offering price for a privatized enterprise.

We denote by $V^{*}$ such a realization of the process for which the new policy is imposed and the investment cost changes from $I_{l}$ to $I_{h}$, where $I_{h}>I_{l}$. $I_{h}$ is assumed to be deterministic. ${ }^{6}$ The firm does not know the value of $V^{*}$ but knows only its cumulative density function, $\Psi\left(V^{*}\right) . \Psi(\cdot)$ is assumed to be truncated normal with mean $\mu$ and variance $\omega^{2} .{ }^{7}$ Consequently, if the investment cost has not increased by time $\tau$, while $\widehat{V}$ is the highest realization of the process so far, the cost will not increase at any $u>\tau$ as long as $V(t) \leq \widehat{V}$

\footnotetext{
${ }^{5}$ If, instead, a downward change in investment cost is considered, the same solution methodology can be applied as in the remainder of the paper. Consequently, a unique realization of the underlying process has to be found for which the marginal cost of waiting beyond the optimal investment threshold equals the benefit of waiting associated with the expected decrease in the investment cost.

${ }^{6} \mathrm{An}$ extension for the stochastic cost would entail replacing $I_{h}$ in the calculations by the expression $\left(\int_{A}{\widetilde{I_{h}}}^{1-\beta} d \Phi\left(\widetilde{I_{h}}\right)\right)^{\frac{1}{1-\beta}}$, where $\Phi\left(\widetilde{I_{h}}\right)$ is the cumulative distribution function of the stochastic investment cost and $A$ is the support of the distribution. Parameter $\beta$ is defined in the main text.

${ }^{7}$ The truncation level is irrelevant as long as it falls into the interval $(0, V(0))$.
} 
for all $t \leq u$. Hence, the probability of the jump in the investment cost is a function of $V$ alone.

In order to restrict our analysis to the most interesting case, we impose the following assumption:

$$
\left(V_{h}-I_{h}\right)\left(\frac{\mu}{V_{h}}\right)^{\beta}<\mu-I_{l}<\frac{1}{\beta-1} I_{l}
$$

where $\beta$ is given by

$$
\beta=-\frac{\alpha}{\sigma^{2}}+\frac{1}{2}+\sqrt{\left(\frac{\alpha}{\sigma^{2}}-\frac{1}{2}\right)^{2}+\frac{2 r}{\sigma^{2}}},
$$

and $V_{h}\left(\equiv \beta I_{h} /(\beta-1)\right)$ is the optimal investment threshold corresponding to the cost $I_{h}$. Assumption (2) states that in the average case it is not optimal to wait with investing until the upward change in cost occurs and that without the expected policy change, the firm would invest optimally at the level of $V$ which exceeds $\mu$.

\subsection{Value of the Investment Opportunity}

Since the value of the project that triggers the increase in the investment cost is not known beforehand, two scenarios are possible. In the first scenario the investment occurs before the change in the investment cost, and in the second scenario the investment takes place after the upward change. Consequently, the value of the investment opportunity reflecting the structure of the expected payoff, has the following form:

$$
\begin{aligned}
F_{s}(V, \widehat{V} \mid I= & \left.I_{l}\right)=p_{s}(\widehat{V}) E\left[\left(V_{s}-I_{l}\right) e^{-r T_{s}}\right]+ \\
& +\left(1-p_{s}(\widehat{V})\right) E\left[\left(V_{h}-I_{h}\right) e^{-r T_{h}}\right]
\end{aligned}
$$

where $p_{s}(\widehat{V})$ is the conditional (on the highest realization of $V, \widehat{V}$ ) probability that the investment cost will not increase before the investment is made optimally, and $T_{s}$ and $T_{h}$ denote the first passage time corresponding to the optimal investment threshold at the low and at the high cost, respectively. After rearranging and including these expectations, we obtain the following maximization problem that allows for finding the optimal investment threshold:

$$
\begin{aligned}
F_{s}\left(V, \widehat{V} \mid I=I_{l}\right)=\max _{V_{s}}\left[\frac{1-\Psi\left(V_{s}\right)}{1-\Psi(\widehat{V})}\left(V_{s}-I_{l}\right)\left(\frac{V}{V_{s}}\right)^{\beta}+\right. \\
\left.+\left(1-\frac{1-\Psi\left(V_{s}\right)}{1-\Psi(\widehat{V})}\right)\left(V_{h}-I_{h}\right)\left(\frac{V}{V_{h}}\right)^{\beta}\right] .
\end{aligned}
$$

$V_{s}$ is the optimal investment threshold in case the investment takes place before the change in cost, and $\widehat{V}$ is the highest realization of the process so far. Hence, $\left(1-\Psi\left(V_{s}\right)\right) /(1-\Psi(\widehat{V}))$ is the probability that the jump in the investment cost will not occur by the moment $V$ is 
equal to $V_{s}$, given that the shock has not occurred for $V$ smaller than $\widehat{V}$. Therefore, equation (5) implies that the value of the investment opportunity is equal to the weighted average of the values of two investment opportunities. They correspond to the investment cost $I_{l}$ and $I_{h}$, respectively, given that the investment is made optimally (at $V_{s}$ if the cost is still equal to $I_{l}$ and at $V_{h}$ if the upward change has already occurred). ${ }^{8}$

The value of the investment opportunity depends on the highest realization of the process, $\widehat{V}$. A higher $\widehat{V}$ (thus a one closer to $V_{s}$ ) implies a lower probability of the costincrease trigger falling into the interval $\left(\widehat{V}, V_{s}\right)$ and, as a consequence, a higher probability of making the investment at the lower cost, $I_{l}$. In order to calculate the value of the investment opportunity, we first need to establish the value of $V_{s}$ by solving the maximization problem.

\subsection{Optimal Investment Threshold}

The optimal investment threshold, $V_{s}$, is found by maximizing the value of the investment opportunity (cf. the RHS of equation (5)).

Proposition 1 Investment is made optimally at $V_{s}$ which is the solution to the following equation:

$$
h\left(V_{s}\right) V_{s}^{2}+(\beta-1) V_{s}-\left(V_{s} h\left(V_{s}\right)+\beta\right) I_{l}-h\left(V_{s}\right) \frac{(\beta-1)^{\beta-1}}{\beta} \frac{V_{s}^{\beta+1}}{I_{h}^{\beta-1}}=0,
$$

where $h(x)=\frac{\psi(x)}{1-\Psi(x)}$ denotes the hazard rate and $\psi(x) \equiv \frac{\partial \Psi(x)}{\partial x}$.

\section{Proof. See Appendix A.}

In our case, the hazard rate has the following interpretation. The probability of the upward change in the investment cost occurring during the nearest increment of the value of the project, $d V$, (given that the cost-increase has not occurred by now) is equal to the appropriate hazard rate multiplied by the size of the value increment, i.e. to $h(V ; \cdot) d V$.

\footnotetext{
${ }^{8} \mathrm{It}$ is worth pointing out that for $I_{h} \rightarrow \infty$ the value of the investment opportunity boils down to:

$$
F_{s}\left(V, \widehat{V} \mid I=I_{l}\right)=\max _{V_{s}}\left[\frac{1-\Psi\left(V_{s}\right)}{1-\Psi(\widehat{V})}\left(V_{s}-I_{l}\right)\left(\frac{V}{V_{s}}\right)^{\beta}\right],
$$

which directly corresponds to the result of Lambrecht and Perraudin (2003). In the other limiting case, i.e. for $I_{h} \rightarrow I_{l}$, the value of investment opportunity reduces to

$$
F_{s}\left(V, \widehat{V} \mid I=I_{l}\right)=\left(V_{l}-I_{l}\right)\left(\frac{V}{V_{l}}\right)^{\beta}
$$

which is the formula obtained by McDonald and Siegel (1986). 


\section{Solution Characteristics}

In this section we analyze how the optimal threshold is affected by changes in the parameters characterizing the dynamics of the project value. In Appendix B we prove that the optimal investment threshold, $V_{s}$, decreases both in $I_{h}$, and in $\beta$, and it increases in $I_{l}$. The former result is intuitive since a threat of a higher future investment cost leads the firm to accept the investment project sooner. The latter two results are consistent with the standard real option theory.

The optimal investment rule depends also on the firm's conjecture about the probability distribution underlying the expected policy change.

\subsection{Hazard Rate}

The hazard rate of the arrival of the cost-increase trigger is one of the basic inputs for calculating the optimal investment threshold. From (8) the following result can be obtained.

Proposition 2 The optimal investment threshold is decreasing in the hazard rate of the costincrease trigger, i.e. the following inequality holds:

$$
\left.\frac{d V_{s}}{d h(V)}\right|_{V=V_{s}}<0
$$

Proof. See Appendix A.

This result implies that an increasing incremental probability of the jump leads to an earlier optimal exercise. The intuition is that an increasing probability of a partial deterioration of the investment opportunity after a small appreciation in the project value decreases the value of waiting. Proposition 2 has a useful implication. A change in a model parameter that affects the hazard rate in one direction, shifts the optimal investment threshold in the opposite side. Consequently, we are able to establish how the investment threshold is affected by changes in the parameters of the distribution function underlying the occurrence of the jump.

\subsection{Trigger Value Uncertainty}

In order to analyze how the optimal investment threshold is affected by the uncertainty of the cost-increase trigger, we only need to establish the sign of the relationship between the hazard rate and this uncertainty. The changes in trigger-value uncertainty are analyzed by applying a mean-preserving spread (see Rotschild and Stiglitz, 1970), which is equivalent to changing the variance of the underlying probability distribution, while keeping the mean fixed. 
If the cost-increase trigger, $V^{*}$, is known with certainty, the investment is made optimally (by assumption (2)) at an infinitesimal instant before $V^{*}$ is reached. At this point, the hazard rate is zero so there is no risk that the cost increases before this trigger is reached. As the uncertainty marginally increases, the hazard rate, for any $V \in[V(0), \mu)$, first increases and then decreases in the mean-preserving spread. ${ }^{9}$ This is a direct consequence of the fact that the probability mass spreads out as uncertainty increases. When uncertainty is low, the probability mass goes up with uncertainty for any $V \in[V(0), \mu)$ and, for sufficiently large uncertainty, this probability mass is negatively related to uncertainty. It turns out that the non-monotonicity feature is the driving force of the results obtained in this paper. An example is shown in Figure 1.

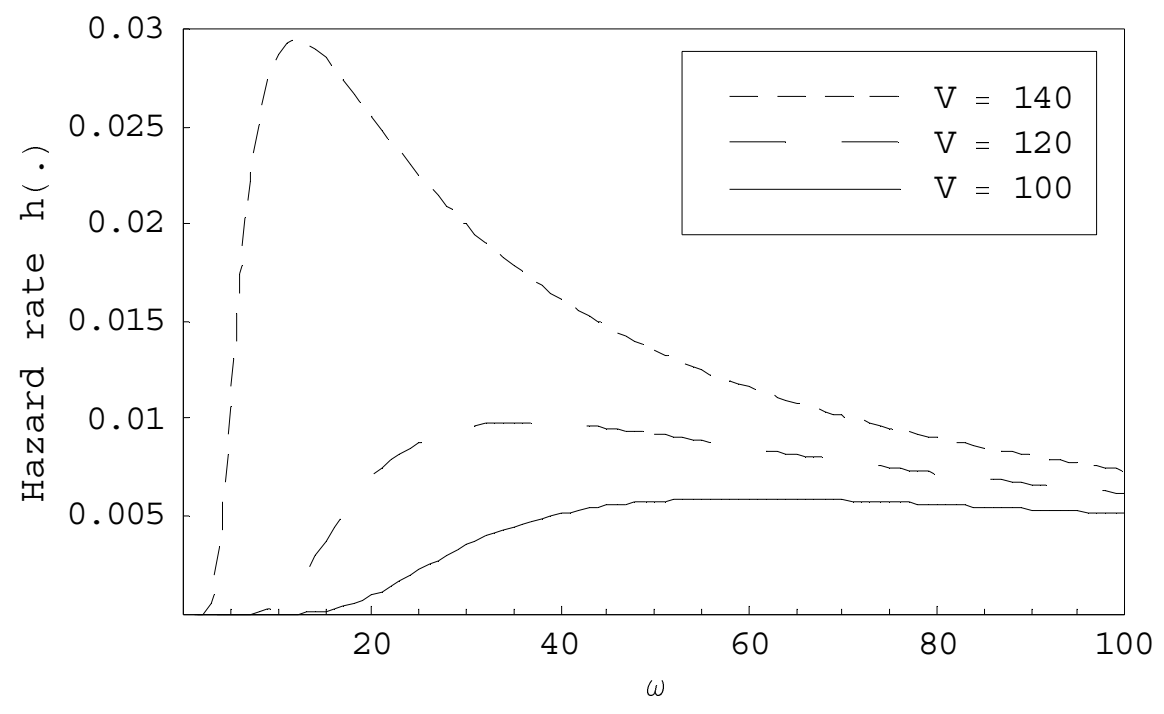

Figure 1: The relationship between the hazard rate and standard deviation calculated for a density function with mean 150. Hazard rates are plotted for $V=100,120$ and 140.

It also holds that for each degree of the trigger value uncertainty, such a value of $V<\mu$, say $\widetilde{V}$, exists such that for $V \in[V(0), \widetilde{V})$ the hazard rate increases, and for $V \in(\widetilde{V}, \mu)$ decreases, in this uncertainty. This form of the relationship between the hazard rate and the uncertainty implies (via Proposition 2) that $V_{s}$ decreases in the uncertainty if it falls into the interval $[V(0), \widetilde{V})$ and increases outside this interval

Consequently, in order to determine the sign of the effect of uncertainty on $V_{s}$, we need to establish the relative position of $V_{s}$ with respect to $\widetilde{V}$. Recall that $\omega$ is the standard deviation of the density function underlying the cost-increase trigger. Since the expression for $V_{s}$ is already known (see (8)), all we have to calculate is $\widetilde{V}$ as a function of $\omega$, such that,

\footnotetext{
${ }^{9}$ It can be shown that this property holds also for other frequently used density functions, such as uniform, exponential and Pareto.
} 
for each pair $(V, \omega)$, the following condition holds:

$$
\left.\frac{\partial h(V)}{\partial \omega}\right|_{V=\tilde{V}}=0
$$

It can be shown that $\widetilde{V}$ decreases with uncertainty. ${ }^{10}$ Consequently, for a relatively low degree of uncertainty, it holds that $V_{s}<\widetilde{V}(<\mu)$. Since for $V<\widetilde{V}$ the hazard rate increases in $\omega, V_{s}$ falls when the uncertainty rises. After the uncertainty reaches a critical level, say $\omega^{e}$, at which $V_{s}=\widetilde{V}$, the hazard rate at $V_{s}$ decreases in $\omega$ and the optimal threshold begins to increase. This implies that the optimal investment threshold attains its minimum for $\omega=\omega^{e}$. Now, we are able to formulate the following proposition.

Proposition 3 There exists a non-monotonic relationship between the optimal investment threshold and the trigger value uncertainty. At a low degree of uncertainty, the marginal increase in uncertainty leads to an earlier optimal investment. The reverse is true for a high degree of uncertainty. There exists a unique $\omega^{e}$, such that $V_{s}\left(\omega^{e}\right)=\widetilde{V}\left(\omega^{e}\right)$, which separates the areas of low and high uncertainty levels.

\section{Proof. See Appendix A.}

At low levels of uncertainty concerning the policy change the firm responds to an increase of this uncertainty by investing earlier (i.e. at a lower $V$ ). This is because the probability of an earlier implementation of the policy change increases. However, when this uncertainty becomes sufficiently high, the firm is more willing to ignore the information about the expected change since the quality of this information has deteriorated too much. The marginal impacts of a higher probability of an early change and of the increased "noisiness" of the firm's conjecture offset exactly at the level of uncertainty equal to $\omega^{e}$.

Figures 2 and 3 show the relationship between the uncertainty, $\omega$, and the optimal investment threshold. In Figure 2 it can be seen that the optimal investment threshold is first decreasing and then increasing in the uncertainty concerning the value of the trigger. The minimum is always reached when $V_{s}(\omega)$ intersects $\tilde{V}(\omega)$. The hazard rate increases in $\omega$ in the area located to the south-west from $\widetilde{V}(\omega)$ and decreases in the north-eastern region. The opposite holds for $V_{s}$. Moreover, the optimal threshold is higher if the expected change in the investment cost is smaller.

In Figure 3 it can be noticed that the point, $\widetilde{V}$, at which the derivative of the hazard rate is equal to zero decreases when the trigger uncertainty increases. As long as $V_{s}<\widetilde{V}$, the optimal threshold also decreases (see the location of $V_{s}^{L}$ ). When the standard deviation is equal to $\omega^{e}, V_{s}$ equals $\widetilde{V}$. After a further increase in the uncertainty, $\widetilde{V}$ continues to decrease

\footnotetext{
${ }^{10}$ Although $\tilde{V}(\omega)$ cannot be written explicitly, its value and the negative dependence on $\omega$ can be easily established numerically.
} 


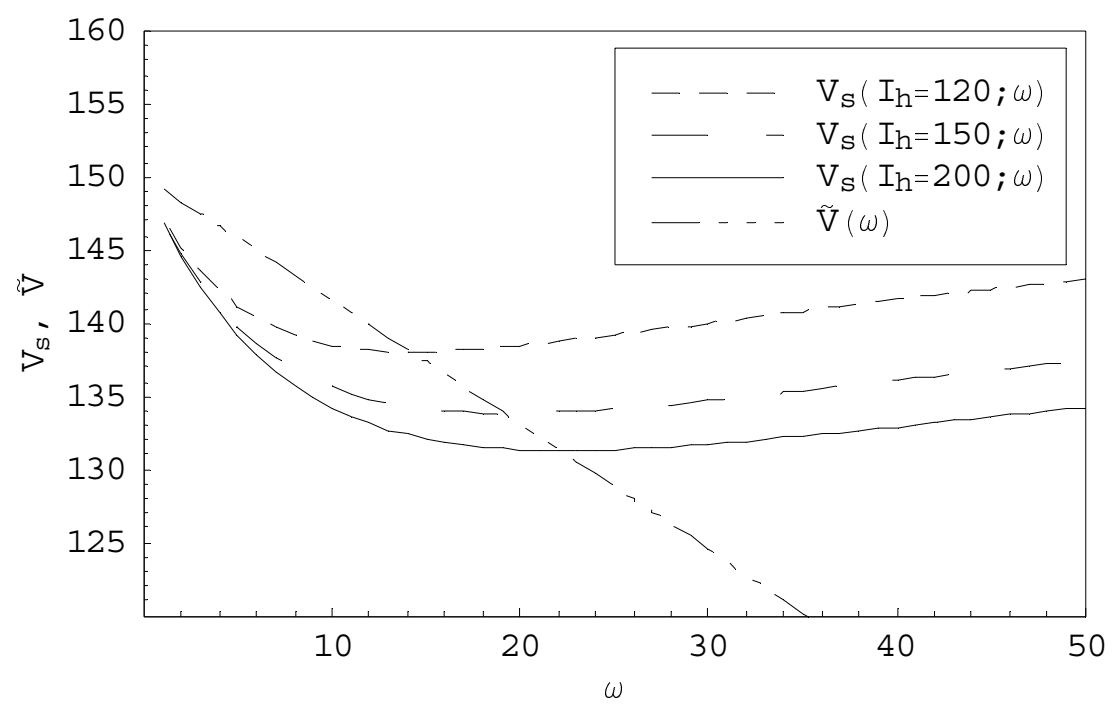

Figure 2: The relationship between the uncertainty, $\omega$, and the optimal investment threshold, $V_{s}$, for different magnitudes of the high investment cost $\left(I_{h}=120,150\right.$ and 200). The values are calculated for a density function with mean 150. The original investment cost $I_{l}$ equals 100. An intersection of $V_{s}$ and $\widetilde{V}$ corresponds to the minimal investment threshold, $V_{s}\left(\omega^{e}\right)$. The parameters of the underlying process are: $\alpha=0, r=0.025$ and $\sigma=0.1$.

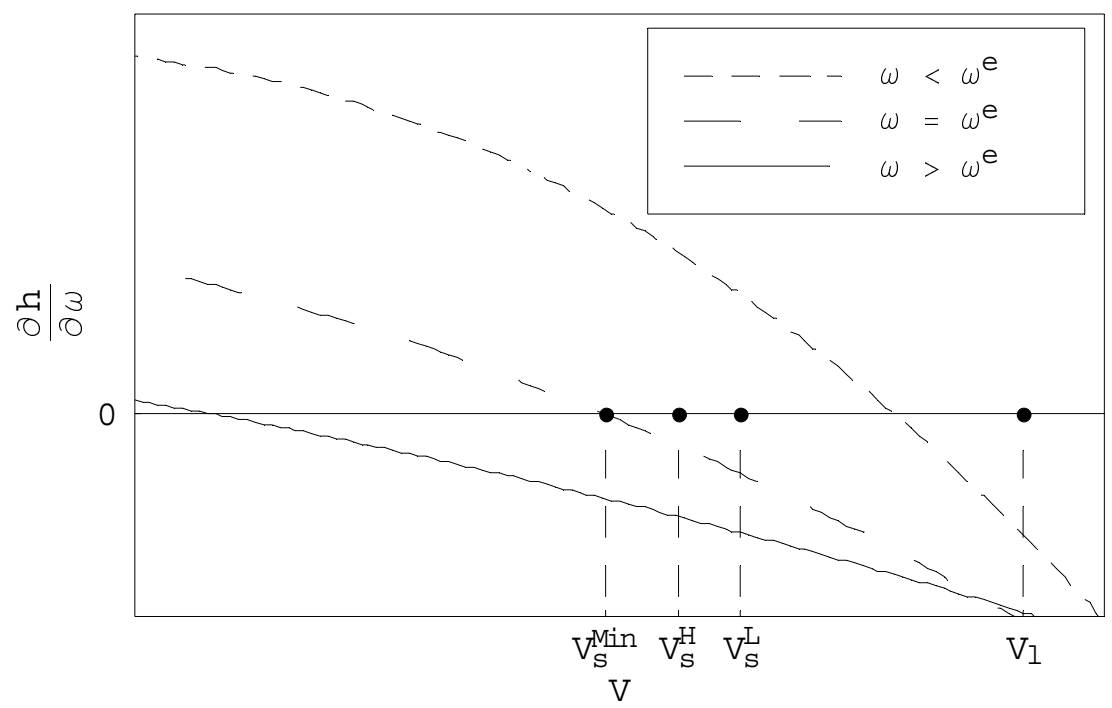

Figure 3: The relationship between $V$ and the derivative of the hazard rate with respect to the trigger value uncertainty. The optimal investment thresholds, $V_{s}^{M i n}, V_{s}^{H}$, and $V_{s}^{L}$, correspond to uncertainty levels equal to, higher than, and lower than the level of uncertainty triggering the earliest investment, respectively. $V_{l}$ is the optimal investment threshold in the absence of the expected policy change. 
and $V_{s}$ starts to increase (see $V_{s}^{H}$ ). For a sufficiently high degree of uncertainty $V_{s}$ tends to the unconditional threshold, denoted by $V_{l}\left(\equiv \beta I_{l} /(\beta-1)\right) .{ }^{11}$

\section{Valuation and Investment Probability}

The policy change uncertainty has direct implications for the value of the investment opportunity and the probability that the investment is made before the change in the investment cost occurs. In Table 1 we provide a numerical illustration made under the assumption that the investment cost still equals $I_{l}$ at $V(0)$.

\begin{tabular}{|c|c|c|c|c|c|}
\hline \multirow{2}{*}{$\frac{\mu=160}{I_{h}}$} & \multicolumn{5}{|c|}{$F(V), P\left(V_{s}<V^{*} \mid V^{*}>V(0)\right)$} \\
\hline & 100 & 50 & 25 & 10 & 5 \\
\hline \multirow[t]{2}{*}{110} & 61.54 & 66.65 & 70.58 & 71.24 & 66.00 \\
\hline & 0.68 & 0.55 & 0.44 & 0.42 & 0.53 \\
\hline \multirow[t]{2}{*}{125} & 55.82 & 57.11 & 56.94 & 53.25 & 48.66 \\
\hline & 0.75 & 0.68 & 0.66 & 0.74 & 0.88 \\
\hline \multirow[t]{2}{*}{150} & 50.93 & 50.01 & 48.28 & 46.28 & 45.27 \\
\hline & 0.80 & 0.78 & 0.80 & 0.87 & 0.95 \\
\hline \multirow[t]{2}{*}{200} & 46.69 & 44.70 & 42.93 & 43.01 & 43.92 \\
\hline & 0.85 & 0.86 & 0.90 & 0.93 & 0.97 \\
\hline \multirow[t]{2}{*}{500} & 42.16 & 40.51 & 40.00 & 40.86 & 42.98 \\
\hline & 0.91 & 0.96 & 1.00 & 0.97 & 0.98 \\
\hline \multirow[t]{2}{*}{$\infty$} & 40.62 & 40.00 & 40.00 & 40.30 & 42.66 \\
\hline & 0.94 & 1.00 & 1.00 & 0.98 & 0.97 \\
\hline$V_{l}=200$ & $\alpha=0.02$ & $\sigma=$ & 0.1 & $r=$ & 0.05 \\
\hline
\end{tabular}

Table 1 . The values of the investment opportunity and probabilities of investing at $I_{l}$ for the following parameter values: investment cost before the jump $I_{l}=100$, investment cost after the jump ranging from 110 to infinity, standard deviation of the probability distribution underlying the policy change ranging from 5 to 100 . The initial value of the process equals $V(0)=\widehat{V}=140$.

Table 1 confirms that the magnitude of the change in the investment cost results in $i$ ) deteriorating the value of the investment opportunity, and $i i$ ) an increased probability of investing before the shock occurs (which is a direct consequence of the lower optimal threshold).

An interesting observation can be made upon analyzing the relationship between the trigger-value uncertainty and the value of the investment opportunity. The non-monotonicity

\footnotetext{
${ }^{11}$ Since it holds that $\lim _{\omega \rightarrow \infty} h\left(V ; \mu, \omega^{2}\right)=0$.
} 
of this relationship results from the interaction of two opposite effects. First, increasing the variance, $\omega^{2}$, implies lower quality of the firm's information about the moment of the policy change. This factor affects the value of the investment opportunity negatively. On the other hand, higher uncertainty reduces the probability mass in the interval $\left[V(0), V_{s}\right]$. This implies that the probability of survival on the interval $\left[V(0), V_{s}\right]$ becomes higher, which enhances the value of the investment opportunity. ${ }^{12}$ It appears that in situations where the magnitude of the change in the investment cost is small, the value of the project is highest for a moderate precision of the conjecture about the timing of the change. Conversely, if the investment opportunity is to deteriorate completely upon the occurrence of the shock, the value of the project is most likely to be equal to its static NPV, i.e. the value of the project minus investment cost, for a moderate precision of the conjecture.

The non-monotonicity of the value of investment opportunity in the variance of the trigger $V^{*}$ does not contradict the standard (real) option theory according to which the relationship between the option value and risk is positive. Option theory describes the relationship between the volatility of the underlying asset $(\sigma)$ and the option value. Here, we analyze the impact of the uncertainty concerning the strike price of the option $(\omega)$ on its value, and option theory does not yield any predictions concerning the sign of this relationship.

\section{Implications for the Investment Credit Tax Policy Change}

To illustrate the design of a credit tax policy change that triggers the earliest investment, we construct the following numerical example. The values of the basic model parameters are as follows: $r=0.05, \alpha=0.02$, and $\sigma=0.125$. Consider a continuum of firms with investment $\operatorname{cost} I_{l}$ distributed uniformly over the interval $[65,105]$. The initial value of the project $V(0)$ is 130 . First, $V^{*}$ is assumed to be known and equal to 147.5. Then the average expected time to invest is 9.37 years. ${ }^{13}$ Using $V^{*}$ as a policy instrument and lowering it to 140 results in the average expected time to invest being shortened to 6.08 years. A further decrease in $V^{*}$ would result in increasing the average expected time to invest, since firms with $I_{l}$ close to 105 would be better off by postponing their investment until their $V_{h}$ (since the first part of condition (2) would be violated for them). For these firms the project value is too small to invest before the policy change occurs.

\footnotetext{
${ }^{12}$ The positive impact on the value of the investment opportunity results from the fact that conditional on $V^{*}>V(0)$ the cumulative density function of $V^{*}$ is decreasing in $\omega$ for sufficiently large $\omega$. This is equivalent, by definition, to the increase of the value of the conditional survival function.

${ }^{13}$ The expected time $E\left[T^{*}\right]$ to hit the barrier $y$ starting from $x$ for the process $(1)$ is given by

$$
E\left[T^{*}\right]=\frac{1}{\alpha-\frac{1}{2} \sigma^{2}} \ln \left(\frac{y}{x}\right) .
$$
}

For a derivation of the probability distribution of the first passage time see Harrison (1985) for a formal exposition and Dixit (1993) for a heuristic approach. 
Alternatively, the authority can leave $V^{*}$ at the initial level of 147.5 and introduce uncertainty about its value. It holds that for $\omega=7$, the optimal investment threshold $V_{s}$ of the firm with the highest investment $\operatorname{cost}\left(I_{l}=105\right)$ is approximately equal to $V^{*}$. Moreover, for $\omega=7$ and $I_{l}=105$, the threshold $V_{s}$ is increasing in $\omega$ (i.e. for $I_{l}=105$ it holds that $\omega=7>\omega^{e}$; cf. also Figures 1 and 2). Consequently, a further increase in $\omega$ would result in the situation where firms with the highest investment cost invest after the occurrence of policy change, which would increase the average expected time to invest. This implies that $\omega=7$ remains the level of uncertainty at which the average expected time to invest is lowest and equal to 4.95 years. Therefore, comparing the two situations allows for the conclusion that abstaining from using uncertainty as a policy instrument results in the average expected time to invest being almost $23 \%$ higher than in the case where uncertainty is used optimally.

\section{Conclusions}

In this paper we consider an investment opportunity of a firm in a situation where the investment cost is irreversible and subject to an increase resulting from a policy change. The value of the cost-increase trigger is unknown to the firm. Instead, the firm has information about the probability distribution characterizing the authority's policy. Furthermore, it is taken into account that a policy change occurs under certain economic conditions.

We show that the threat of a policy change resulting in a higher investment cost leads to a reduction in the option value of waiting. Consequently, the firm invests earlier than in the case of a constant investment cost. The optimal investment threshold decreases in the magnitude of the change in investment cost and increases in market volatility (the latter result also holds in the standard real option framework). Moreover, we show that the value of the investment opportunity is non-monotonic in the magnitude of policy change uncertainty.

Our main result is that the impact of trigger value uncertainty on the optimal investment threshold is non-monotonic. If the uncertainty is sufficiently low, then the investment threshold is negatively related to the trigger value uncertainty. However, a rise in the un-

certainty beyond a certain critical point reverses this relationship and leads to an increase of the optimal investment threshold. We illustrate our results with an example of the optimal design of the authority's policy change, where the authority's aim is to accelerate investment undertaken by firms. It is shown that the shortest average expected time to invest is associated with a strictly positive level of the policy change uncertainty. 


\section{A Proofs of Propositions}

Proof of Proposition 1. The implicit solution for the optimal investment threshold is

found by calculating the first order condition of (5). By differentiating (5) with respect to $V_{s}$, and equalizing to zero, we obtain:

$$
\begin{aligned}
& \frac{V^{\beta}}{V_{s}^{\beta+1}}\left(V_{s}-\beta V_{s}+\beta I_{l}\right) \frac{1-\Psi\left(V_{s}\right)}{1-\Psi(\widehat{V})}-\left(V_{s}-I_{l}\right)\left(\frac{V}{V_{s}}\right)^{\beta}\left(\frac{\psi\left(V_{s}\right)}{1-\Psi(\widehat{V})}\right) \\
& +\left(V_{h}-I_{h}\right)\left(\frac{V}{V_{h}}\right)^{\beta} \frac{\psi\left(V_{s}\right)}{1-\Psi(\widehat{V})}=0 .
\end{aligned}
$$

Further simplification yields:

$$
\begin{aligned}
& \frac{1}{V_{s}^{\beta+1}}\left(V_{s}-\beta V_{s}+\beta I_{l}\right)\left(1-\Psi\left(V_{s}\right)\right)-\left(V_{s}-I_{l}\right)\left(\frac{1}{V_{s}}\right)^{\beta} \psi\left(V_{s}\right) \\
& +\left(V_{h}-I_{h}\right)\left(\frac{1}{V_{h}}\right)^{\beta} \psi\left(V_{s}\right)=0,
\end{aligned}
$$

thus

$$
-(\beta-1) V_{s}+\beta I_{l}-h\left(V_{s}\right)\left(V_{s}-I_{l}\right) V_{s}+\left(V_{h}-I_{h}\right) V_{s}^{\beta+1}\left(\frac{1}{V_{h}}\right)^{\beta} h\left(V_{s}\right)=0 .
$$

Since $V_{h}=\frac{\beta}{\beta-1} I_{h}$ (after the jump the McDonald-Siegel problem is left), this is equal to

$$
-h\left(V_{s}\right) V_{s}^{2}-(\beta-1) V_{s}+\left(h\left(V_{s}\right) V_{s}+\beta\right) I_{l}+V_{s}^{\beta+1} h\left(V_{s}\right) \frac{I_{h}}{\beta-1}\left(\frac{\beta-1}{\beta I_{h}}\right)^{\beta}=0,
$$

what in a straightforward way leads to (8).

In order to prove that (8) is the expression for the maximal value of the project, we calculate the second order condition, which is equal to the following derivative:

$$
\frac{\partial}{\partial V_{s}}\left(-h\left(V_{s}\right) V_{s}^{2}-(\beta-1) V_{s}+\left(V_{s} h\left(V_{s}\right)+\beta\right) I_{l}+h\left(V_{s}\right) \frac{(\beta-1)^{\beta-1}}{\beta^{\beta}} \frac{V_{s}^{\beta+1}}{I_{h}^{\beta-1}}\right) .
$$

After differentiating, we obtain expression for the second order condition of (5):

$$
\begin{aligned}
\frac{\partial^{2} F_{s}}{\partial V_{s}^{2}}= & -\left(h^{\prime}\left(V_{s}\right) V_{s}+h\left(V_{s}\right)\right)\left(V_{s}-I_{l}-\frac{V_{s}}{\beta}\left(\frac{\beta-1}{\beta} \frac{V_{s}}{I_{h}}\right)^{\beta-1}\right) \\
& -h\left(V_{s}\right) V_{s}\left(1-\left(\frac{\beta-1}{\beta} \frac{V_{s}}{I_{h}}\right)^{\beta-1}\right)-(\beta-1) .
\end{aligned}
$$

The sign of the second component is negative since

$$
1-\left(\frac{\beta-1}{\beta} \frac{V_{s}}{I_{h}}\right)^{\beta-1}>1-\left(\frac{\beta-1}{\beta} \frac{V_{h}}{I_{h}}\right)^{\beta-1}=0 .
$$


The sign of the first component can be determined by notifying that the lower bound of $V_{s}$, denoted by $\underline{V_{s}}$, is a solution to the following equation, cf. (2)):

$$
\underline{V_{s}}-I_{l}=\left(V_{h}-I_{h}\right)\left(\frac{V_{s}}{\overline{V_{h}}}\right)^{\beta}
$$

For $V_{s}=\underline{V_{s}}$ the second factor in the first component of (A.2) is equal to zero and for $V_{s}>\underline{V_{s}}$ it is positive. Therefore the whole expression is negative as long as $\left(h^{\prime}\left(V_{s}\right) V_{s}+h\left(V_{s}\right)\right)$ his positive. The latter is straightforward to show since $V_{s}$ and $h\left(V_{s}\right)$ are always positive and, on the basis of assumption (2) implying that $V_{s}<\mu$, the hazard rate increases in $V$.

Proof of Proposition 2. By differentiating (B.1) with respect to the hazard rate, while taking into account that $V_{h}=\frac{\beta}{\beta-1} I_{h}$, we obtain:

$$
\frac{\partial H}{\partial h}=V_{s}\left(V_{s}-I_{l}-\left(V_{h}-I_{h}\right)\left(\frac{V_{s}}{V_{h}}\right)^{\beta}\right)>0 .
$$

The inequality holds since the both factors are positive (cf. (A.4)). Since $\frac{\partial H}{\partial V_{s}}$ is also positive, we directly obtain the sign of (9).

Proof of Proposition 3. Using the result of Proposition 2 about the inverse relationship between the hazard rate and the optimal investment threshold, we still have to prove that the sign of the derivative

$$
\left.\frac{\partial h\left(x ; \mu, \omega^{2}\right)}{\partial\left(\omega^{2}\right)}\right|_{x=V}
$$

is positive for $\omega \in\left(0, \omega^{*}\right)$ and negative for $\omega \in\left(\omega^{*}, \infty\right)$ for any $V$ smaller than the mean $\mu$. First, we recall that

$$
h(x)=\frac{e^{-\frac{(x-\mu)^{2}}{2 \omega^{2}}}}{\int_{x}^{\infty} e^{-\frac{(y-\mu)^{2}}{2 \omega^{2}}} d y} .
$$

Using chain rule we obtain

$$
\frac{\partial h(x)}{\partial\left(\omega^{2}\right)}=\Theta\left[(x-\mu)^{2} \int_{x}^{\infty} e^{-\frac{(y-\mu)^{2}}{2 \omega^{2}}} d y-\int_{x}^{\infty}(y-\mu)^{2} e^{-\frac{(y-\mu)^{2}}{2 \omega^{2}}} d y\right],
$$

where $\Theta$ is given by

$$
\Theta \equiv \frac{\frac{1}{2 \omega^{4}} e^{-\frac{(x-\mu)^{2}}{2 \omega^{2}}}}{\left(\int_{x}^{\infty} e^{-\frac{(y-\mu)^{2}}{2 \omega^{2}}} d y\right)^{2}}>0
$$

Let us define

$$
u \equiv x-\mu \text {. }
$$


After substituting (A.10) into the second factor of (A.8) and working out the integrals we obtain

$$
\begin{aligned}
\frac{\partial h(x)}{\partial\left(\omega^{2}\right)}= & \frac{\Theta \omega \sqrt{\pi}}{\sqrt{2}}\left[u^{2}+\omega^{2}\left(\sqrt{\frac{2 u^{2}}{\pi \omega^{2}}} e^{-\frac{u^{2}}{2 \omega^{2}}}-1\right)+\right. \\
& \left.+\left(u^{2}-\omega^{2}\right) \operatorname{erf}\left(\frac{|u|}{\sqrt{2} \omega}\right)\right]
\end{aligned}
$$

where

$$
\operatorname{erf}(z) \equiv \frac{2}{\sqrt{\pi}} \int_{0}^{z} e^{-y^{2}} d y
$$

is the error function. It holds that the first component in brackets of (A.11) is a positive constant. To prove that $\frac{\partial h(x)}{\partial\left(\omega^{2}\right)}$ is positive for $\omega \in\left(0, \omega^{*}\right)$ and negative for $\omega \in\left(\omega^{*}, \infty\right)$ we show that the second component of (A.11) is decreasing from zero with the limit in minus infinity, and that the third component initially positive and globally decreasing.

It is sufficient to show that

$$
\Gamma \equiv \omega\left(\sqrt{\frac{2 u^{2}}{\pi \omega^{2}}} e^{-\frac{u^{2}}{2 \omega^{2}}}-1\right)
$$

is negative and decreasing with the limit in minus infinity to conclude that the second component of (A.11) possesses these properties, too (since it is an increasing transformation of (A.13)). First, we can immediately observe that $\lim _{\omega \rightarrow 0} \Gamma=0$. Moreover, it holds that

$$
\frac{\partial \Gamma}{\partial \omega}=\sqrt{\frac{2}{\pi}} e^{-\frac{u^{2}}{2 \omega^{2}}} \frac{\left|u^{3}\right|}{\omega^{3}}-1
$$

which is always negative since the maximum of $\frac{\partial \Gamma}{\partial \omega}$ (which occurs for $\omega=\sqrt{3}|u|$ ) equals $\sqrt{\frac{54}{\pi}} e^{-\frac{3}{2}}-1<0$. Therefore $\Gamma$ is globally decreasing. Furthermore, it is straightforward to show that

$$
\lim _{\omega \rightarrow \infty} \Gamma=-\infty .
$$

Finally, we decompose the third component in brackets in (A.11) into

$$
u^{2} \operatorname{erf}\left(\frac{|u|}{\sqrt{2} \omega}\right)-\omega^{2} \operatorname{erf}\left(\frac{|u|}{\sqrt{2} \omega}\right)
$$

and substitute

$$
z \equiv \frac{|u|}{\sqrt{2} \omega}
$$

Clearly, the first part of (A.16) is always positive and decreasing. To show that its second part is decreasing as well, we calculate the sign of its derivative

$$
\frac{\partial\left(-\omega^{2} \operatorname{erf}(z)\right)}{\partial \omega}=-\frac{4 \omega}{\sqrt{\pi}}\left[\int_{0}^{z}\left(e^{-y^{2}}-e^{-z^{2}}\right) d y\right]<0,
$$

since $e^{-y^{2}}$ is a decreasing function of $y$. This completes the proof. 


\section{B Comparative Statics}

Let us define the LHS of (8) as a function:

$$
\begin{aligned}
& H\left(V_{s}, I_{l}, I_{h}, \beta\right) \\
= & h\left(V_{s}\right) V_{s}^{2}+(\beta-1) V_{s}-\left(V_{s} h\left(V_{s}\right)+\beta\right) I_{l}-h\left(V_{s}\right) \frac{(\beta-1)^{\beta-1}}{\beta^{\beta}} \frac{V_{s}^{\beta+1}}{I_{h}^{\beta-1}} .
\end{aligned}
$$

Differentiating (B.1) with respect to $I_{l}, I_{h}$ and $\beta$, respectively, yields:

$$
\begin{aligned}
& \frac{\partial H}{\partial I_{l}}=-\left(V_{s} h\left(V_{s}\right)+\beta\right)<0, \\
& \frac{\partial H}{\partial I_{h}}=(\beta-1) h\left(V_{s}\right) \frac{(\beta-1)^{\beta-1}}{\beta^{\beta}} \frac{V_{s}^{\beta+1}}{I_{H}^{\beta}}>0, \\
& \frac{\partial H}{\partial \beta}=V_{s}-I_{l}-h\left(V_{s}\right) \frac{(\beta-1)^{\beta-1}}{\beta^{\beta}} \frac{V_{s}^{\beta+1}}{I_{h}^{\beta-1}} \ln \left(\frac{\beta-1}{\beta} \frac{V_{s}}{I_{h}}\right)>0,
\end{aligned}
$$

$\forall I_{l}, I_{h}$ satisfying $0<I_{l}<I_{h}, \forall \beta \in(1, r / \alpha)$ if $\alpha>0$ and $\forall \beta \in(1, \infty)$ if $\alpha \leq 0$. Furthermore, differentiating (B.1) with respect to $V_{s}$ gives:

$$
\begin{aligned}
\frac{\partial H}{\partial V_{s}}= & \left(h^{\prime}\left(V_{s}\right) V_{s}+h\left(V_{s}\right)\right)\left(V_{s}-I_{l}-\left(V_{h}-I_{h}\right)\left(\frac{V_{s}}{V_{h}}\right)^{\beta}\right)+ \\
& +h\left(V_{s}\right) V_{s}\left(1-\left(\frac{\beta-1}{\beta} \frac{V_{s}}{I_{h}}\right)^{\beta-1}\right)+(\beta-1) .
\end{aligned}
$$

From the proof of Proposition 1 (cf. (A.4)) it is known that $\frac{\partial H}{\partial V_{s}}$ is positive. Finally, by observing that

$$
\frac{d V_{s}}{d z}=-\frac{\frac{\partial H}{\partial z}}{\frac{\partial H}{\partial V_{s}}},
$$

where $z$ is an arbitrary parameter of our interest, we know that

$$
\operatorname{sgn} \frac{d V_{s}}{d z}=-\operatorname{sgn} \frac{\partial H}{\partial z},
$$

what completes the analysis.

\section{References}

Altug, Sumru, Fanny S. Demers, and Michel Demers (2001). The Impact of Tax Risk and Persistence on Investment Decisions, Economics Bulletin, 5, 1-6.

Berrada, Tony (1999). Valuing Real Option When Time to Maturity Is Uncertain, Working Paper, University of Geneva.

Dixit, Avinash (1993). The Art of Smooth Pasting, Vol. 55 in Fundamentals of Pure and Applied Economics, Harwood Academic Publishers. 
Dixit, Avinash, And Robert Pindyck (1996). Investment under Uncertainty (2nd printing), Princeton University Press.

Harrison, Michael J. (1985). Brownian Motion and Stochastic Flow Systems, John Wiley \& Sons.

Hassett, Kevin A., and Gilbert E. Metcalf (1999). Investment with Uncertain Tax Policy: Does Random Tax Policy Discourage Investment?, Economic Journal, 109, 372393.

Lambrecht, Bart, and William Perraudin (2003). Real Options and Preemption under Incomplete Information, Working Paper, Birkbeck College.

Lander, Diane M., and George E. Pinches (1998). Challenges to the Practical Implementation of Modeling and Valuing Real Options, Quarterly Review of Economics and Finance, 38, 537-567.

McDonald, Robert, and Daniel Siegel (1986). The Value of Waiting to Invest, Quarterly Journal of Economics, 101, 707-728.

Panteghini, Paolo M., and Carlo Scarpa (2003). Irreversible Investment and Regulatory Risk, CES-IFO Working Paper 934.

Rotschild, Michael, And Joseph E. Stiglitz (1970). Increasing Risk: I. A Definition, Journal of Economic Theory, 2, 225-243.

Schwartz, Eduardo S. And Mark Moon (2000). Evaluating Research and Development Investments, In: Project Flexibility, Agency and Competition: New Developments in the Theory and Applications of Real Options, Michael J. Brennan and Lenos Trigeorgis (eds.), Oxford University Press, 85-106.

Schwartz, Eduardo S. and Lenos Trigeorgis (eds.) (2001). Real Options and Investment under Uncertainty, MIT Press. 